\title{
Irradiated mesenchymal stem cells support stemness maintenance of hepatocellular carcinoma stem cells through Wnt/ $\beta$-catenin signaling pathway
}

\author{
Jing Hou ${ }^{1}$, Naping Zhao ${ }^{2}$, Pengxi Zhu ${ }^{3}$, Jun Chang ${ }^{1}$, Yan Du and Wei Shen ${ }^{1 *}$
}

\begin{abstract}
Background: Cancer stem cells are the main reason of relapse, metastasis and resistance to anti-cancer therapies of Hepatocellular carcinoma (HCC). Mesenchymal stem cells (MSCs) are an important part of the tumor microenvironment. MSCs have been demonstrated to be involved in drug resistance in tumor. How MSCs contribute to radiotherapy resistance of HCC is still indistinct.

Methods: Flow cytometry analysis was performed to isolate CD133+ cells from HCC cell lines Huh7 and PLC. The stemness of Huh7-CD133 and PLC-CD133 those were co-cultured with IR-MSCs were investigated by Colony formation assay. Tumor formation in nude mice was used to explore the tumorigenicity of CD133+ cancer cells. The activating Wnt/ $\beta$-catenin signaling pathway in CSCS were also detected by RT-PCR and Western blotting.

Results: We report that irradiated MSCs (IR-MSCs) could increase the ratio of CD133+ cells in hepatocellular carcinoma cells. IR-MSCs could promote stemness maintenance of HCC stem cells. After co-cultured with IR-MSCs, liver cancer stem cells (CSCs) presented increased colony formation ability and tumor formation ability. We also found IR-MSCs promoted Wnt expression of CSCs. Reverse suppression experiment showed that when Wnt inhibitor was added into the culture medium, the effect of IR-MSCs on stemness maintenance was counteracted.
\end{abstract}

Conclusions: These data showed that IR-MSCs could support stemness maintenance of CSCs by activating Wnt/Bcatenin signaling pathway.

Keywords: Mesenchymal stem cells, Liver cancer stem cells, Tumor microenvironment, Wnt/ $\beta$-catenin signaling pathway

\section{Background}

HCC is one of the most common malignant tumors in the world. It is easy to metastasize and relapse, and seriously endangers human life. High expression of multidrug resistance genes in liver cancer results in insensitive

\footnotetext{
*Correspondence: joanshenwei@126.com

${ }^{1}$ GCP Office, Xinhua Hospital Affiliated to Shanghai Jiaotong University School of Medicine, 1665 Kongjiang Road, Shanghai 200092, People's Republic of China

Full list of author information is available at the end of the article
}

to chemotherapy. For patients with liver cancer who have lost their chance of surgery, radiation therapy has gradually become an important treatment for primary liver cancer. However, some patients are still not sensitive to radiotherapy, so enhance the radiotherapy sensitivity and effective treatment of liver cancer are particularly urgent and important.

In the study of tumor origin, CSCs have become a hotspot and attract more and more attention. With the discovery of subpopulations with the characteristics of CSCs in liver cancer cell lines, more and more evidence

c) The Author(s) 2020. This article is licensed under a Creative Commons Attribution 4.0 International License, which permits use, sharing, adaptation, distribution and reproduction in any medium or format, as long as you give appropriate credit to the original author(s) and the source, provide a link to the Creative Commons licence, and indicate if changes were made. The images or other third party material in this article are included in the article's Creative Commons licence, unless indicated otherwise in a credit line to the material. If material is not included in the article's Creative Commons licence and your intended use is not permitted by statutory regulation or exceeds the permitted use, you will need to obtain permission directly from the copyright holder. To view a copy of this licence, visit http://creativeco mmons.org/licenses/by/4.0/. The Creative Commons Public Domain Dedication waiver (http://creativecommons.org/publicdomain/ zero/1.0/) applies to the data made available in this article, unless otherwise stated in a credit line to the data. 
indicates that liver cancer stem cells are the main cause of liver cancer $[1,2]$.The characteristics of CSCs are very similar to those of normal stem cells, such as self-renew and multi-directional differentiation. And maybe only a small number of such cells control tumor growth [3]. Numerous studies confirm that tumor occurrence, development, metastasis and recurrence and chemotherapy resistance are closely related to CSCs $[4,5]$. The insensitivity of liver CSCs to radiotherapy is also the main cause of insensitivity of HCC to radiotherapy. By using CD133 and other surface markers, the researchers isolated a population of stem cell-like tumor cells from liver cancer cell lines and liver cancer tissues that showed similar proliferation, self-renewal and differentiation capabilities to stem cells, also showed strong tumor formation ability in nude mice. The growth of liver CSCs is inseparable from the support of liver cancer microenvironment. How does the liver cancer microenvironment affect the stemness of CSCs?

With the development of molecular biology technology, it has been found that the tumor microenvironment plays an important role in tumor evolution, while MSCs are an important adult stem cells that constitutes the tumor microenvironment. MSCs have the characteristics of supporting hematopoiesis, immune regulation and multi-directional differentiation, and can also migrate to damaged tissues, chronic inflammatory reaction sites and repair damaged tissues [6]. Tumors have been described as non-healable lesions, and a large number of studies have shown that MSCs have tumor tropism. After MSCs homing to tumor tissue, how does it work on tumor cells? In some animal tumor models.

Exogenous MSCs can promote melanoma, colon cancer, multiple myeloma, lung cancer, and glioblastoma Progression of tumor [7-9]. How MSCs promote resistance of radiotherapy of CSCs need a further study. In this study, we isolated CSCs from HCC cell lines by using CD133 marker. We found that irradiated MSCs (IR-MSCs) could promote stemness maintenance of liver CSCs. After co-cultured with IR-MSCs, CSCs presented increased colony formation capability and tumor formation in nude mice.

Wnt/ $\beta$-catenin signaling pathway is a highly conserved pathway in biological evolution, and is regulated by a series of small proteins inside and outside the cell. It has been known to play an important role in embryonic cell development, proliferation, transformation, cell adhesion, cell survival and apoptosis [10]. In recent years, with the gradual expansion of research on Wnt signaling pathway, it has been found that signaling molecules of Wnt pathway are involved in the process of tumors stem cell self-renewal regulation [11, 12].In normal breast cells, the expression of Wnt signaling pathway-related proteins is restricted, and its expression is increased in breast cancer cells, suggesting that Wnt signaling pathway is responsible for the maintenance of breast cancer stem cells [13]. Wnt signaling pathway also plays an important role in the self-renewal and differentiation of liver CSCs. In a further study, we found that IR-MSCs could activate Wnt signaling pathway. In addition, Wnt inhibitor counteracted the effect of IR-MSCs on stemness maintenance of liver CSCs. Thus, the data showed that IR-MSCs supported maintaining stemness of CSCs through Wnt $/ \beta$ catenin signaling pathway.

\section{Results}

\section{IR-MSCs increased proportion of CD133 positive cells} in hepatocellular carcinoma cells

Umbilical cord mesenchymal stem cells were presents from Dr. Shi in the Institute of health sciences. MSCs were irradiated by 6 Gy and then recovered for $12 \mathrm{~h}$. Hepatocellular carcinoma cells Huh7 and PLC were cocultured with IR-MSCs or control MSCs for 7 days. Then CD133 was detected on the surface of Huh7 and PLC by flowcytometry. As shown in Fig. 1a, b, after co-culture with MSCs, the proportion of CD133 positive cells did not change significantly. Whereas, compared with MSCs group, the proportion of CD133 positive cells was increased significantly after co-culture with IR-MSCs. In addition, western blotting and RT-PCR results showed that IR-MSCs promoted expression of CD133 in Huh7 and PLC both at mRNA level and protein level (Fig. 1c, d).

\section{IR-MSCs promoted stemness maintenance of CD133 positive cells}

To investigate the effect of IR-MSCs on stemness maintenance of hepatocellular carcinoma cells, we isolated $\mathrm{CD}_{133^{+}}$cells from Huh7 and PLC as tumor stem cells, then $\mathrm{CD}_{133^{+}}$cells were co-cultured with IR-MSCs. 7 days later, Huh7-CD133 and PLC-CD133 were plated into 6 well plate as 500 cells per well. Then colony was stained by crystal violet and counted 7 days after culture. In addition, we detected tumorigenesis of tumor stem cells. $1 \times 10^{6}$ Huh7-CD133 and PLC-CD133 were injected into nude mice subcutaneously after co-culture with IR-MSCs for 7 days. 4 weeks later, tumor volume and weight were tested in different groups. As shown in Fig. 2c, IR-MSCs could promoted tumor formation of tumor stem cells at a great degree. As shown in Fig. 2d, e, tumor volume and tumor weight was increased after cocultured with IR-MSCs.

\section{IR-MSCs promoted Wnt signaling pathway}

Wnt/ $\beta$-catenin signaling pathway play a key role in the process of tumors stem cell self-renewal regulation 

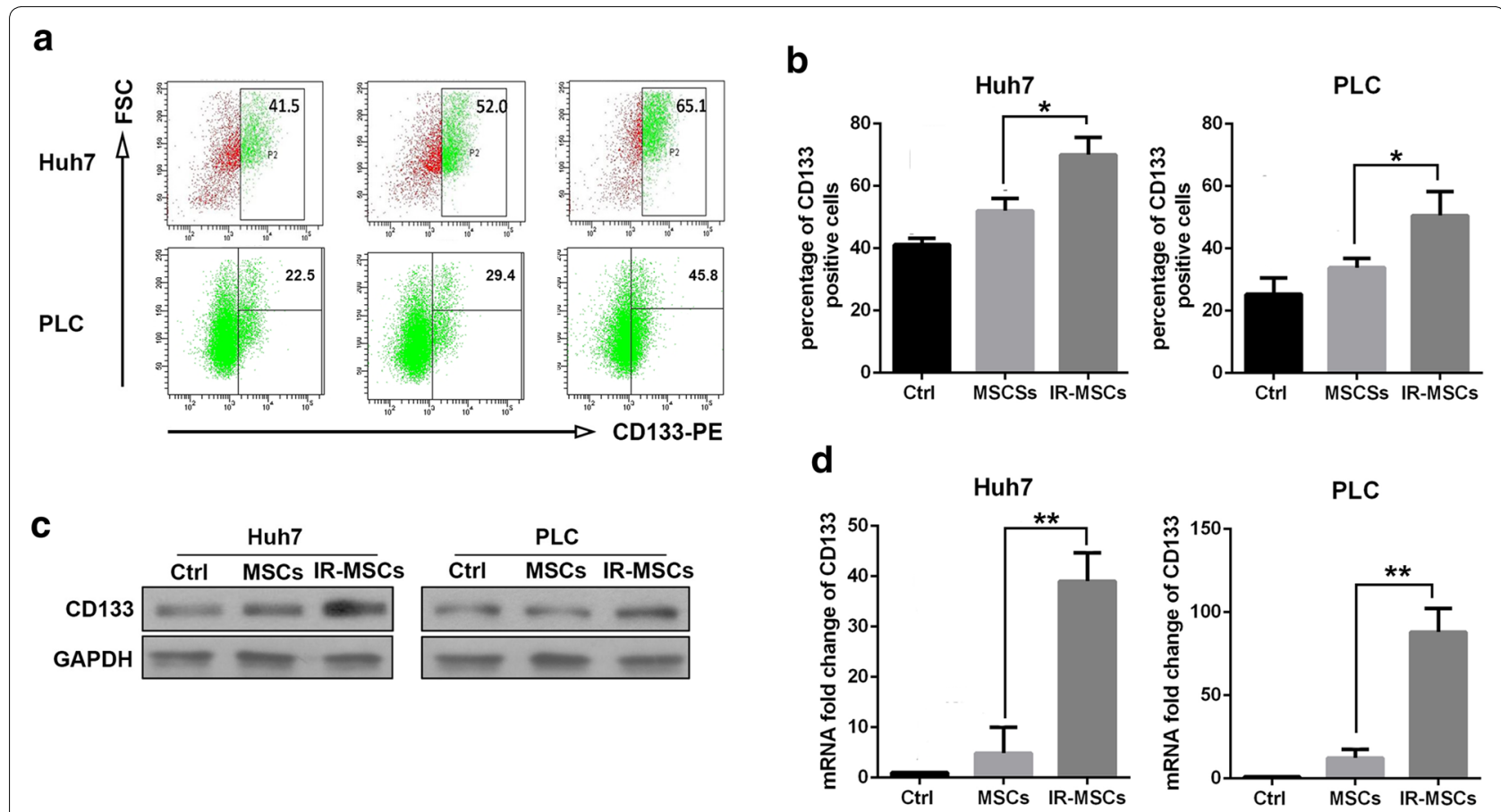

Fig. 1 IR-MSCs increased proportion of CD133 positive cells in hepatocellular carcinoma cells. CD133 detection in Huh7 and PLC after CO-culture with MSCs and IR-MSCs for 7 days. a Detection of CD133 positive cells proportion in HCC cell lines Huh7 and PLC by flow cytometry. $\mathbf{b}$ Quantification of panel A. c Protein expression of CD133 detected by western blotting assay. $\mathbf{d}$ mRNA expression of CD133 detected by RT-PCR. IR-MSCs, irradiated MSCS

[11]. Thus, we detected Wnt and $\beta$-catenin expression in tumor stem cells. We detected the gene expression of Wnt family members by Real-Time PCR. The data showed the expression of Wnt3a in IR-MSCs group was higher than MSCs group(Additional file 1: Fig. S1).As shown in Fig. 3a, after co-culture with IR-MSCs, both Wnt and $\beta$-catenin were increased in Huh7-CD133 and PLC-CD133. Data in Fig. 3b also showed the same result at mRNA level.

\section{Inhibition of Wnt signaling pathway could suppress the function of IR-MSCs}

To verify the role of Wnt/ $\beta$-catenin signaling pathway in stemness maintenance of tumor stem cells, we used inhibitor of Wnt signal, PRI-724. Huh7-CD133 was treated by PRI-724(30 nM)for $48 \mathrm{~h}$, then co-cultured with IR-MSCs. We found that PRI-724 could reverse the enhanced effect of IR-MSCs on CD133 expression, colony formation and tumorigenesis in nude mice of tumor stem cells (Fig. 4).

\section{Discussion}

Radiotherapy resistance of HCC is becoming more and more common which affects therapy of HCC patients. While, the mechanism of HCC radiotherapy resistance is not clear. Increasing evidence indicated that CSCs were the main reason of radiotherapy resistance. CSCs describe a class of stem cells capable of self-renewal and multi-differentiation [14-17] which can maintain the proliferation and growth of tumors and evade endogenous and exogenous regulation of the stability of their internal environment. These characteristics of what is known as "stemness" allow for the growth of the primary cancer tumor as well as the development of new tumors, metastasis and recurrence of tumors $[18,19]$. CSCs can survive while normal cancer cells die under radiotherapy. CD133 has been reported to be a marker of CSCs in many tumors, such as breast, prostate, colon, glioma, liver, lung, ovary cancer [20]. Numerous factors, including the central role of the tumor microenvironment, have been hypothesized to contribute to CSC treatment resistance [21].

Mesenchymal stem cells are a group of adult stem cells which can migrate to tissue injury sites and tumor sites. MSCs have been reported to be very important in tissue injury repair and tumor formation [6]. In former study, we found that MSCs could maintain their stemness after radiotherapy through autophagy activation [22]. In this study, we isolated CSCs from HCC cell lines Huh7 and PLC by using CD133 positive screening and co-cultured 


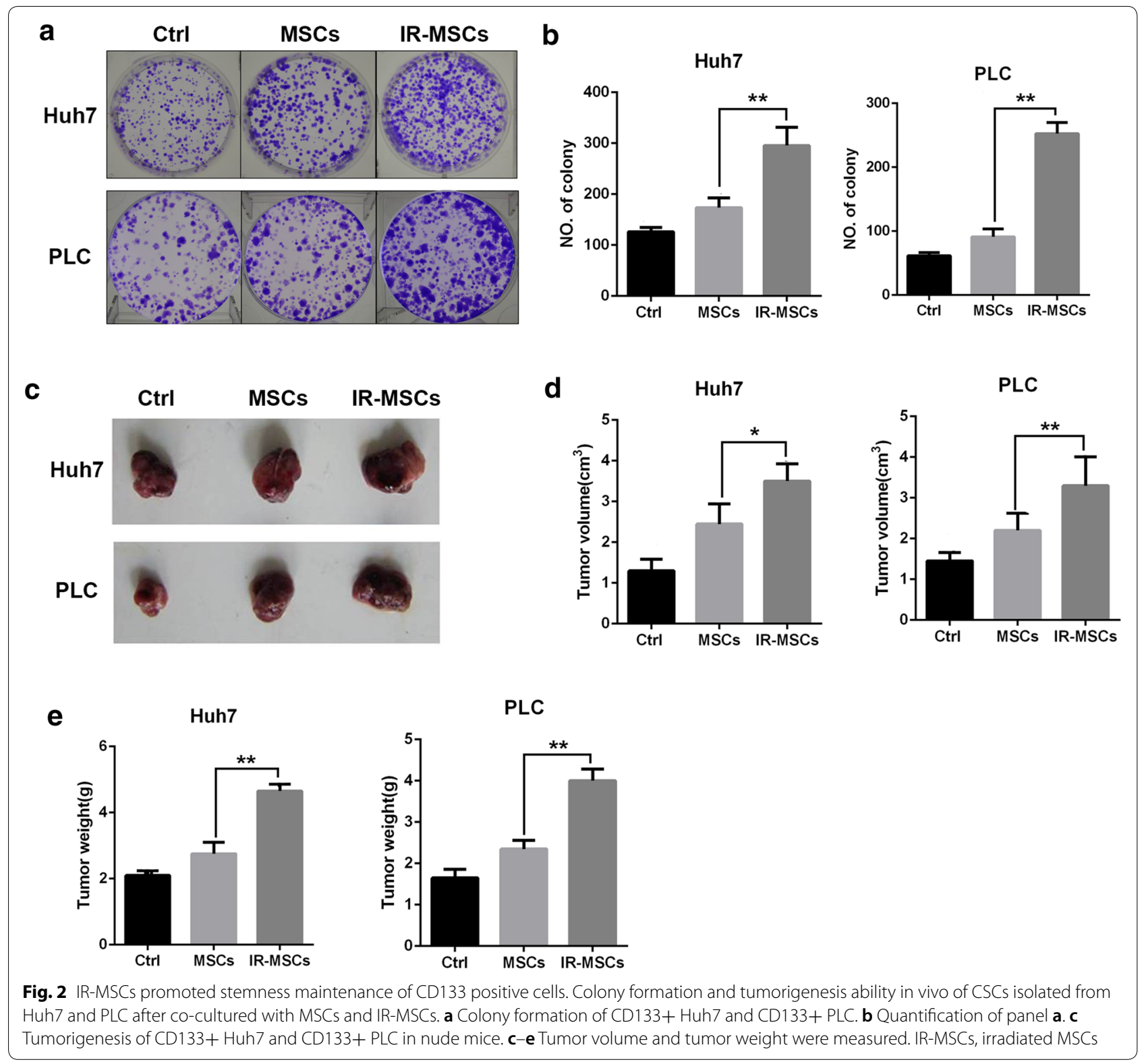

MSCs with CSCs. We found that compared with normal MSCs, irradiated MSCs could support stemness maintenance of cancer stem cells. Expression of stem cell markers, ability of colony formation and tumorigenesis in vivo were increased after co-cultured with IR-MSCs.

Wnt/ $\beta$-catenin signaling pathway has been known to play an important role in embryonic cell development, proliferation, transformation, cell adhesion, cell survival and apoptosis [10]. In recent years, with the gradual expansion of research on Wnt signaling pathway, it has been found that signaling molecules of Wnt pathway are involved in the process of tumor stem cell selfrenewal regulation [11]. We found that IR-MSCs could upregulate Wnt and $\beta$-catenin expression. In order to verify the role of Wnt signaling pathway in stemness maintenance of CSCs, we used Wnt signal inhibitor PRI-724 to pre-treat CSCs to block Wnt signaling pathway in CSCs. Data showed that the effect of stemness support of IR-MSCs was inhibited after PRI-724 pretreatment. Thus, IR-MSCs may support stemness maintenance of CSCs through activating $\mathrm{Wnt} / \beta$-catenin expression.

However, what happens to MSCs after irradiation also needs our thinking. Recently, some studies found non-coding RNAs can active Wnt signaling in cancer stem cells. 

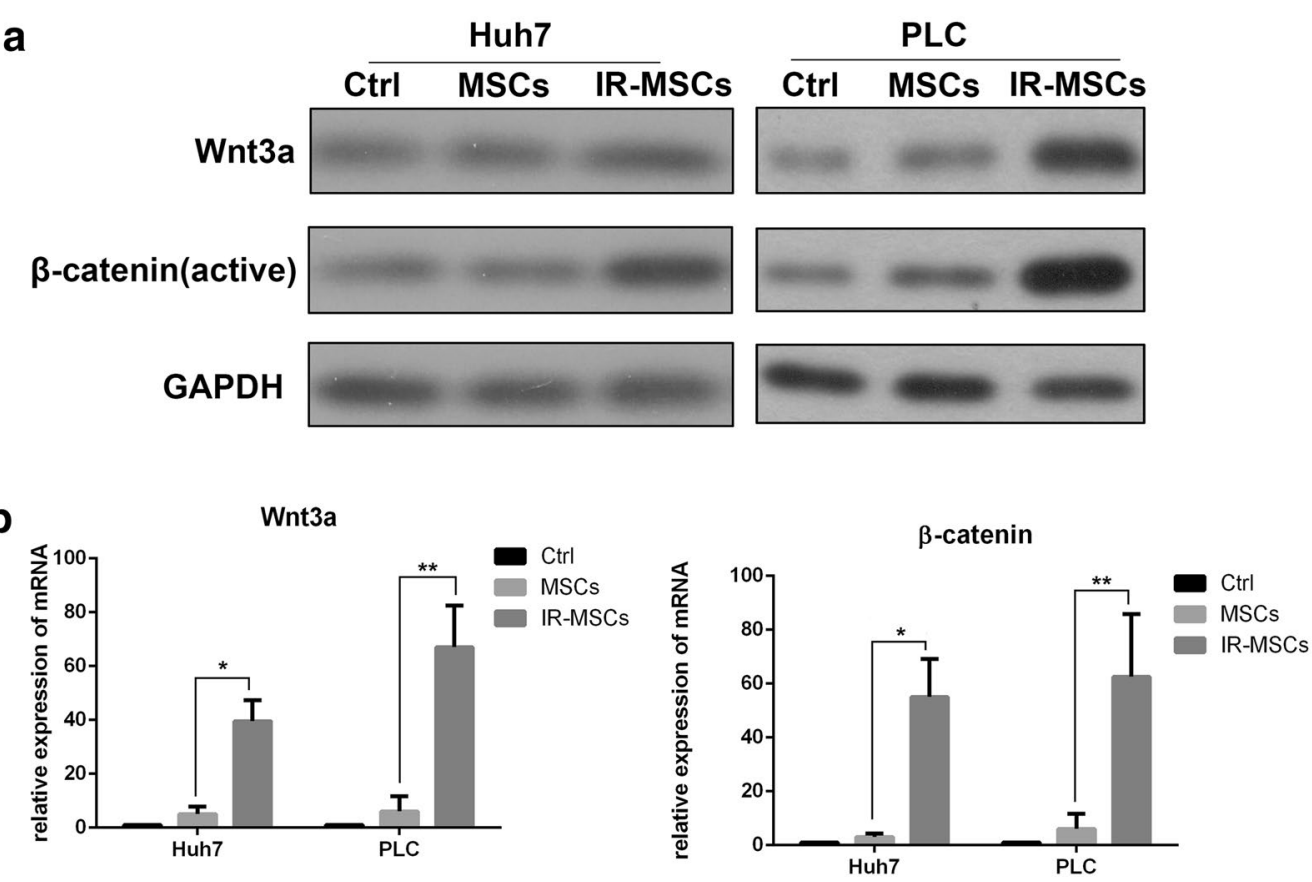

Fig. 3 IR-MSCs promoted Wnt signaling pathway. a Wnt3a and $\beta$-catenin expression in CD133+ Huh7 and CD133+ PLC after co-cultured with MSCs and IR-MSCs. b mRNA expression of Wnt3a and $\beta$-catenin in CD133+ Huh7 and CD133+ PLC

In mammary stem cells, miR-142 recruits the APC mRNA for degradation and increases Wnt signaling [23]. The long non-coding RNA lncTCF7, which is expressed in liver CSCs, was found to activate Wnt signaling is associated with an increased self-renewal capacity of liver CSCs [24]. The other studies showed multiple cytokines exist in tumor microenvironment $[25,26]$, such as transforming growth factor (TGF-), epidermal growth factor (EGF), Insulin-like growth factor (IGF), vascular endothelial growth factor (VEGF) drive cross-talk between tumor cells and stromal cells. What factor is secreted by IR-MSCs to affect the stemness maintenance of CSCs still needs a further study.

\section{Conclusions}

Our data showed that IR-MSCs could support stemness maintenance of CSCs by activating $\mathrm{Wnt} / \beta$-catenin signaling pathway.

\section{Materials and methods Irradiation}

MSCs were plated into T25 plates. MSCs were irradiated with ELEKTA Synergy Linear Accelerator (Cravoley, UK) at $6 \mathrm{~Gy}$ (a dose rate of $350 \mathrm{cGy} / \mathrm{min}$ ) over an appropriate field size when they were $80 \%$ confluent. Irradiated cells were allowed to recover for $12 \mathrm{~h}$.
Isolation of CD133+ populations with flow cytometry

To isolate CD133+ cells from HCC cell lines Huh7 and PLC, both cells were suspended as single cell suspension, then labeled with PE-conjugated anti-CD133 antibody for $30 \mathrm{~min}$, then CD133+ cells were isolated apart from CD133- cells by flow cytometry. They were named as Huh7-CD133 and PLC-CD133, respectively.

\section{Co-culture assay}

Transwell assay was used to perform co-culture assay. Huh7 and PLC were plated in 6-well plate at a density of $1 \mathrm{X} 10^{5}$, MSCs or IR-MSCs were seeded in transwell insert $(0.4 \mu \mathrm{m}$ pore), after co-culture for seven days, Huh7 and PLC were used for other experiments.

\section{CD133 detection with flow cytometry}

HCC cell lines Huh7 and PLC were co-cultured with IR-MSCs for 7 days, then the expression of CD133 was measured by flow cytometry. Cells were labeled with PE-conjugated anti-CD133 antibody, the percentage of CD133+ cancer cells was measured by MACSQuant Analyzer10 flow cytometric system (Miltenyi Biotech, Germany).

\section{Colony formation assay}

Huh7-CD133 and PLC-CD133 were co-cultured with IR-MSCs for 7 days, then were seeded into 6 well plate 


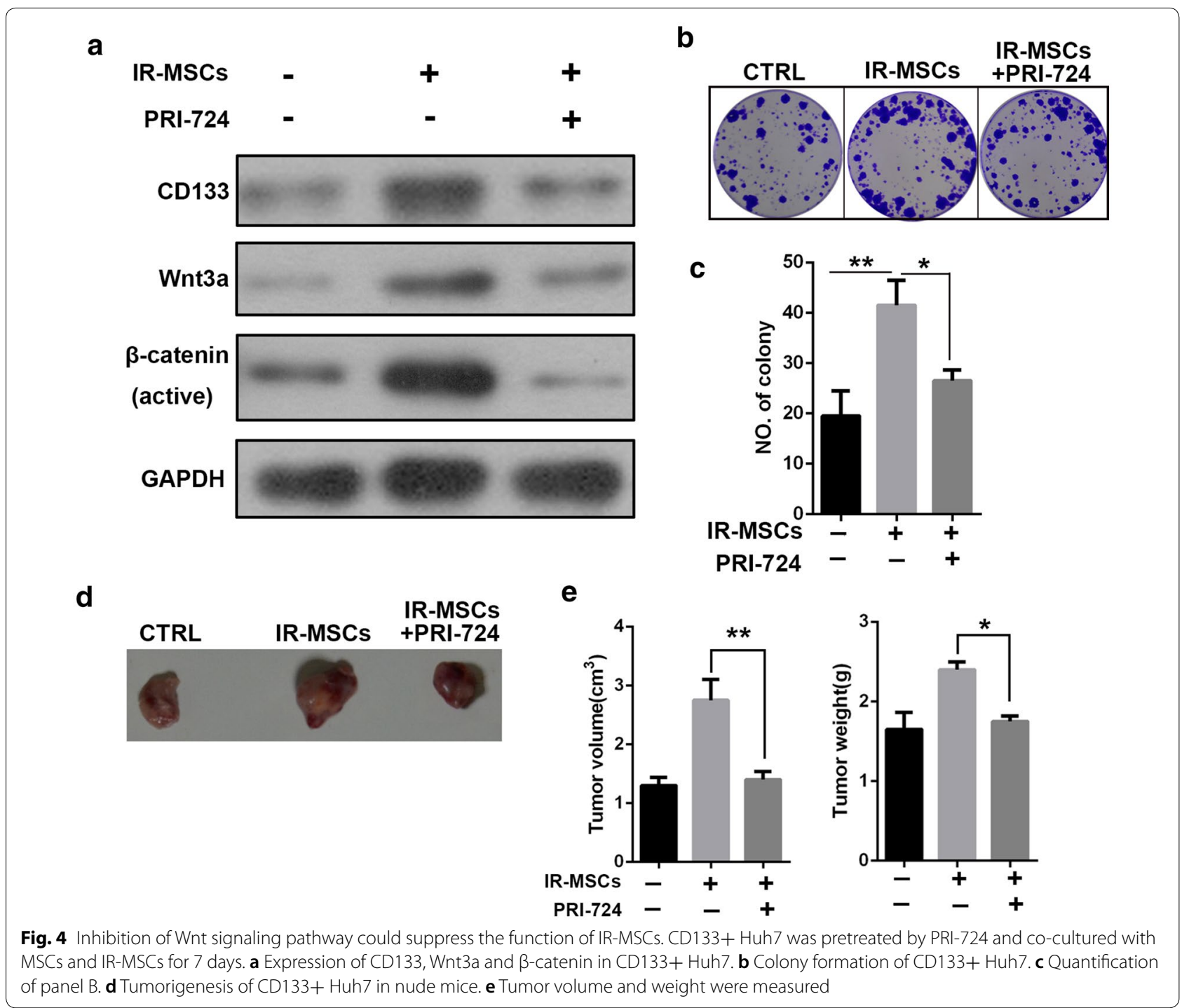

as 500 cells per well. 7 days later, cells were fixed and stained by $0.1 \%$ crystal violet solution. The number of colonies larger than $2 \mathrm{~mm}$ in diameter was counted.

\section{Tumor formation in nude mice}

Six-week-old male athymic BALB/c nu/nu mice were obtained from Shanghai Experimental Animal Center, Chinese Academy of Science. Mice were maintained under a pathogen-free condition and treated in accordance with the institutional animal welfare guidelines. For tumorigenicity assay, Huh7-CD133 and PLC-CD133 were co-cultured with IR-MSCs for 7 days. $1 \times 10^{6}$ cells in $100 \mu \mathrm{L}$ were injected subcutaneously to the left back of mice. Mice were sacrificed 4 weeks after injection. Tumors were collected and volume and weight of tumors were calculated.

\section{RT-PCR}

Total RNA was abstracted by Trizol assay and reversetranscripted to cDNA by bestar qPCR RT kit. mRNA expression was detected by RT-PCR by using bestar real-time PCR master mix with an ABI Prism 7300 system. The primers are as follows: CD133, sense, AGTCGG AAACTGGCAGATAGC, antisense, GGTAGTGTTGTA CTGGGCCAAT; Wnt3a, sense, AGCTACCCGATC TGGTGGTC, antisense, CAAACTCGATGTCCTCGC TAC; $\beta$-catenin, sense, AGCTTCCAGACACGCTAT CAT, antisense, CGGTACAACGAGCTGTTTCTAC; GAPDH, sense, GGAGCGAGATCCCTCCAAAAT, antisense, GGCTGTTGTCATACTTCTCATGG. The primers for genes of Wnt family (Additional file 2: Table S1).

\section{Western blotting}

Total protein was acquired by RIPA and quantified by BCA assay. Proteins of the same mass were tested for 
expression of CD133 (64326, 1:1000,Cell signal technology) and Wnt3a (ab28472, 1:1000, abcam), $\beta$-catenin (ab6302, 1:1000, abcam), GAPDH(ab8245, 1:5000, abcam)by SDS-PAGE, GAPDH was used as control. The assay was performed according to protocols described before [27].

\section{Statistical analysis}

All the experiments were performed 4 times. Student T-test was done to analysis the difference between different groups. ${ }^{*} \mathrm{P}<0.05$ and ${ }^{* *} \mathrm{P}<0.01$ represent a statistical difference. Data were expressed as the mean \pm standard deviation (SD).

\section{Supplementary information}

Supplementary information accompanies this paper at https://doi. org/10.1186/s13578-020-00449-5.

Additional file 1: Figure S1. Gene expression of Wnt family. Huh7 and PLC were co-cultured with ctrl MSCs or IR-MSCs for seven days, then mRNA was abstracted and RT-PCR assay was performed to detect expression of Wnt1,Wnt2, Wnt3, Wnt3a, Wnt4, Wnt5a, Wnt6, Wnt7b, Wnt10b,and Wnt11.

Additional file 2: Table S1. RT-PCR Primers for genes of Wnt family.

\section{Acknowledgements}

Not applicable.

\section{Authors' contributions}

$J H, N Z, P Z, J C, Y D$ and WS conceived and designed the study. JH wrote the manuscript. JC and YD helped to proofread the manuscript. NZ and PZ helped to perform the experiment. WS helped to modify this article. All authors read and approved the final manuscript.

\section{Funding}

This project was supported by National Key R\&D Program of China (Grant No. 2018YFA0107500) and National Science Foundation of China (Grant No: 81402454).

\section{Ethics approval and consent to participate}

The study was carried out strictly according to recommendations from the Guide for the Care and Use of Laboratory Animals of the National Institutes of Health.

\section{Consent for publication}

Not applicable.

\section{Competing interests}

The authors declare that they have no competing interest.

\section{Author details}

${ }^{1}$ GCP Office, Xinhua Hospital Affiliated to Shanghai Jiaotong University School of Medicine, 1665 Kongjiang Road, Shanghai 200092, People's Republic of China. ${ }^{2}$ Department of Pharmacy, Changhai Hospital, Naval Military Medical University, 168 Changhai Road, Shanghai 200433, China. ${ }^{3}$ Department of Pharmacy, Naval Military Medical University, 800 Xiangyin Road, Shanghai 200433, China.

Received: 7 April 2020 Accepted: 19 June 2020

Published online: 03 August 2020

\section{References}

1. Singh SK, et al. Identification of a cancer stem cell in human brain tumors. Cancer Res. 2003;63(18):5821-8.

2. Singh SK, et al. Identification of human brain tumour initiating cells. Nature. 2004;432(7015):396-401.

3. Al-Hajj M, Clarke MF. Self-renewal and solid tumor stem cells. Oncogene. 2004:23(43):7274-82

4. Soltanian S, Matin MM. Cancer stem cells and cancer therapy. Tumour Biol. 2011:32(3):425-40.

5. O'Connor ML, et al. Cancer stem cells: a contentious hypothesis now moving forward. Cancer Lett. 2014;344(2):180-7.

6. Wei $X$, et al. Mesenchymal stem cells: a new trend for cell therapy. Acta Pharmacol Sin. 2013;34(6):747-54.

7. Xu S, et al. Bone marrow-derived mesenchymal stromal cells are attracted by multiple myeloma cell-produced chemokine CCL25 and favor myeloma cell growth in vitro and in vivo. Stem Cells. 2012;30(2):266-79.

8. Barcellos-de-Souza P, et al. Tumor microenvironment: bone marrowmesenchymal stem cells as key players. Biochim Biophys Acta. 2013;1836(2):321-35.

9. Gottschling $\mathrm{S}$, et al. Mesenchymal stem cells in non-small cell lung cancer-different from others? Insights from comparative molecular and functional analyses. Lung Cancer. 2013;80(1):19-29.

10. Nelson WJ, Nusse R. Convergence of Wnt, beta-catenin, and cadherin pathways. Science. 2004;303(5663):1483-7.

11. Reya T, Clevers H. Wnt signalling in stem cells and cancer. Nature. 2005;434(7035):843-50.

12. De Ferrari GV, et al. Wnt/beta-catenin signaling in Alzheimer's disease CNS Neurol Disord Drug Targets. 2014;13(5):745-54.

13. Prosperi JR, Goss KH. A Wnt-ow of opportunity: targeting the Wnt/betacatenin pathway in breast cancer. Curr Drug Targets. 2010;11(9):1074-88.

14. Beck B, Blanpain C. Unravelling cancer stem cell potential. Nat Rev Cancer. 2013;13(10):727-38

15. Reya T, et al. Stem cells, cancer, and cancer stem cells. Nature. 2001;414(6859):105-11.

16. Dean M, Fojo T, Bates S. Tumour stem cells and drug resistance. Nat Rev Cancer. 2005:5(4):275-84

17. Aderetti DA, et al. The hypoxic peri-arteriolar glioma stem cell niche, an integrated concept of five types of niches in human glioblastoma. Biochim Biophys Acta Rev Cancer. 2018;1869(2):346-54.

18. Ajani JA, et al. Cancer stem cells: the promise and the potential. Semin Oncol. 2015;42(Suppl 1):S3-17.

19. Chen $\mathrm{K}$, Huang $\mathrm{YH}$, Chen J. Understanding and targeting cancer stem cells: therapeutic implications and challenges. Acta Pharmacol Sin. 2013;34(6):732-40.

20. Steinbichler TB, et al. Therapy resistance mediated by cancer stem cells. Semin Cancer Biol. 2018:53:156-67.

21. Borovski T, et al. Cancer stem cell niche: the place to be. Cancer Res. 2011;71(3):634-9.

22. Hou J, et al. Autophagy prevents irradiation injury and maintains stemness through decreasing ROS generation in mesenchymal stem cells. Cell Death Dis. 2013:4:e844.

23. Isobe T, et al. miR-142 regulates the tumorigenicity of human breast cancer stem cells through the canonical WNT signaling pathway. Elife. 2014;3:e01977.

24. Wang Y, et al. The long noncoding RNA IncTCF7 promotes self-renewal of human liver cancer stem cells through activation of Wnt signaling. Cell Stem Cell. 2015;16(4):413-25.

25. Doherty MR, et al. Cancer stem cell plasticity drives therapeutic resistance. Cancers. 2016;8(1):8.

26. Vermeulen $L$, et al. Wnt activity defines colon cancer stem cells and is regulated by the microenvironment. Nat Cell Biol. 2010;12(5):468-76.

27. Jin S, et al. Mesenchymal stem cells with enhanced Bcl-2 expression promote liver recovery in a rat model of hepatic cirrhosis. Cell Physiol Biochem. 2016:40(5):1117-28.

\section{Publisher's Note}

Springer Nature remains neutral with regard to jurisdictional claims in published maps and institutional affiliations. 